\title{
Is Breast Implant Associated Autoimmune Disorders Independent of Silicone?
}

\author{
Lily Suh, Connor Drake, Imran Khan, Colby R Neumann, Aladdin H Hassanein, Mithun Sinha
}

Department of Surgery, Indiana University School of Medicine

Background: Breast implant illness (BII) is a systemic autoimmune complication associated with breast implants. Breast implants are used for cosmetic augmentation, reconstructive surgery postbreast cancer treatment, or for prophylactic mastectomy. Thus, these implants are important for quality of life of the subjects. Through this work, we sought to characterize breast implant associated autoimmune disorders and discuss possible causes for its etiology.

Methods: The PubMed and OVID databases were interrogated from 1990-2020 using a query strategy including search term combinations of "implants", "breast implant illness", "autoimmune", "systemic illness".

Results: BII includes a spectrum of autoimmune symptoms such as fatigue, myalgias/arthralgias, dry eyes/mouth, and rash. Review of epidemiological studies in the past thirty years exhibited evidence affirming an association between breast implants and autoimmune diseases. The most commonly recognized were Sjogren's, rheumatoid arthritis, systemic sclerosis, and Raynaud's syndrome. Explantation resulted in alleviation of symptoms in over $50 \%$ patients, strengthening the hypothesis associating breast implants to these problems. Studies have shown silicone is a biologically inert material and unlikely to be the cause of these symptoms. This is supported by the fact that increased risk of autoimmune disease was also reported in patients with other implantable biomaterials such as orthopedic implants. Recent studies conducted by our group and other researchers shed light on a possible role of bacterial biofilm and subsequent hostpathogen interactions as a confounding factor to this problem.

Conclusion: BII could be dependent on biofilm infection and the microenvironment around the implants. Should explantation of the implant continue to be the gold standard for treatment, or should more research be done to treat the underlying cause? The true pathophysiology behind these complaints must be further investigated so that accurate treatment regimens other than explantation can be developed. Translational significance of these studies is not limited to breast implants but extends to other implants as well. 